\title{
INTEGRATION OF COMPLEX VECTOR FIELDS ${ }^{1,2}$
}

\author{
BY J. J. KOHN
}

1. The problem. First we describe the local problem. Let $L_{1}, \ldots, L_{m}$ be vector fields defined in a neighborhood $U$ of the origin in $\boldsymbol{R}^{n}$ by the expressions :

$$
L_{k}=\sum_{j=1}^{n} a_{k}^{j} \frac{\partial}{\partial x_{j}}, \quad k=1, \ldots, m,
$$

where the $a_{k}^{j}$ are infinitely differentiable complex-valued functions on $U$. The local problem is to "solve" the equations

$$
L_{k} u=f_{k}, \quad k=1, \ldots, m .
$$

That is, given functions $f_{1}, \ldots, f_{m}$ we wish to find conditions for the existence of a function $u$ satisfying (1.2); further we wish to describe the set of functions satisfying (1.2) and also their dependence on the $f_{k}$, especially with respect to regularity properties. First consider the homogeneous case when $f_{k}=0$, i.e.,

$$
L_{k} u=0, \quad k=1, \ldots, m .
$$

Any function $u$ satisfying all the above equations must also satisfy the equations

$$
\left[L_{k}, L_{h}\right] u=L_{k} L_{h} u-L_{h} L_{k} u=0 .
$$

Thus it is reasonable to assume that the space spanned by the vector fields $L_{1}, \ldots, L_{m}$ is closed under the bracket operation.

Condition A. This condition is satisfied if

$$
\left[L_{k}, L_{h}\right]=\sum_{j} a_{k h}^{j} L_{j}
$$

where the $a_{k h}^{j} \in C^{\infty}(U)$.

From (1.2) we obtain

$$
\left[L_{k}, L_{h}\right] u=L_{k} f_{h}-L_{h} f_{k}
$$

and hence (1.5) yields

$$
L_{k} f_{h}-L_{h} f_{k}=\sum_{j} a_{k h}^{j} f_{j}, \quad 1 \leqq k<h \leqq m .
$$

\footnotetext{
${ }^{1}$ Somewhat amplified version of the lecture presented at the 673rd meeting of the American Mathematical Society in New York City on March 28, 1970; received by the editors June 28, 1971.

AMS 1969 subject classifications. Primary 3548, 3530.

Key words and phrases. Vector field, differential operator, differential complex, complex manifold, hypoelliptic, bundle, adjoint.

${ }_{2}$ Research supported in part by an NSF project at Princeton University.
} 
Thus we have established the following lemma.

1.7 Lemma. If $L_{1}, \ldots, L_{m}$ satisfy condition $\mathrm{A}$ and if there exists a function $u$ satisfying (1.2) then $f_{1}, \ldots, f_{m}$ satisfy the system (1.6).

Another obvious necessary condition is given in the following.

1.8 Lemma. If (1.2) is satisfied in $U$ and if $P \in U$ and

$$
\sum_{k} c^{k} a_{k}^{j}(P)=0, \text { for } j=1, \ldots, n,
$$

with $c^{k} \in C$ then it follows that

$$
\sum c^{k} f_{k}(P)=0 .
$$

We will assume the following condition which implies (1.9).

Condition B. This condition is satisfied if $L_{1}, \ldots, L_{m}$ are linearly independent at each point, i.e. if the matrix $a_{k}^{j}$ is of rank $m$ at each point.

As will be seen later condition B is extremely restrictive but as yet it has not been possible to weaken it.

The global problem may be formulated as follows. Let $C T(U)$ denote the complexified tangent bundle over $U$ and let $\boldsymbol{L}$ be a complex subbundle of $C T(U)$ with fiber of complex dimension $m$. Condition A can now be replaced by requiring that if $L, L^{\prime} \in \Gamma(L, V)$ (i.e. sections of $L$ over $V \subset U$ ) then

$$
\left[L, L^{\prime}\right] \in \Gamma(\boldsymbol{L}, V) .
$$

We denote by $\boldsymbol{L}^{*}$ the dual bundle of $\boldsymbol{L}$ and for every open $V \subset U$ we define the map

$$
\mathscr{D}: C^{\infty}(V) \rightarrow \Gamma\left(\boldsymbol{L}^{*}, V\right)
$$

if $u \in C^{\infty}(V), \mathscr{D} u$ is determined by setting

$$
\langle\mathscr{D} u, L\rangle=L(u)
$$

for all $L \in \Gamma(L, V)$ where $\langle$,$\rangle denotes the pairings between the fibers of L^{*}$ and $\boldsymbol{L}$. Equation (1.2) can now be written as follows:

$$
\mathscr{D} u=f
$$

where $u \in C^{\infty}(U)$ and $f \in \Gamma\left(\boldsymbol{L}^{*}, U\right)$. Choosing $L_{1}, \ldots, L_{m}$ to be a local basis for $\Gamma(\boldsymbol{L}, U)$ and $\omega^{1}, \ldots, \omega^{m}$ to be the dual bases of $\Gamma\left(\boldsymbol{L}^{*}, U\right)$, it is clear that (1.2) expresses (1.12) in terms of this local basis. The advantage of the above formulation is that it makes sense whenever $U$ is a $C^{\infty}$ manifold. In order to reformulate Lemma 1.7 in those terms, let $L^{*} \wedge L^{*}$ denote the 
skew symmetric product of $L^{*}$ with itself. We then have the natural isomorphism $L^{*} \wedge L^{*} \approx(L \wedge L)^{*}$. Define the $\operatorname{map} \mathscr{D}: \Gamma\left(L^{*}, V\right) \rightarrow \Gamma\left(L^{*} \wedge L^{*}, V\right)$ by setting

$$
\left\langle\mathscr{D} f, L \wedge L^{\prime}\right\rangle=L\left(\left\langle L^{\prime}, f\right\rangle\right)-L^{\prime}(\langle L, f\rangle)-\left\langle\left[L, L^{\prime}\right], f\right\rangle,
$$

where $f \in \Gamma\left(L^{*}, V\right)$ and $L, L^{\prime} \in \Gamma(L, V)$; the symbol $\langle$,$\rangle denotes the pairing$ between the fibers of $\boldsymbol{L} \wedge \boldsymbol{L}$ and $L^{*} \wedge L^{*}$. Thus we obtain the complex

$$
C_{0}^{\infty}(V) \stackrel{\mathscr{Q}}{\rightarrow} \Gamma\left(L^{*}, V\right) \stackrel{\mathscr{Q}}{\rightarrow} \Gamma\left(L^{*} \wedge L^{*}, V\right) ;
$$

it is easily checked that

$$
\mathscr{D}^{2}=0 \text {. }
$$

Lemma 1.7 can now be reformulated as follows. A necessary condition for the existence of $u$ satisfying (1.12) is

$$
\mathscr{D} f=0 .
$$

2. Examples. The following are examples of manifolds $M$ on which we distinguish a subbundle $L$ of the complexified tangent bundle $C T$, which satisfies conditions A and B and we discuss the corresponding local and global problems of solving (1.12).

(I) $M$ is any differentiable manifold and $\boldsymbol{L}=\boldsymbol{C T}$. In this case $\mathscr{D}$ is the exterior derivative (usually denoted by $d$ ). The solution of the local problem is classical, usually called the Poincare lemma, and the global problem can be analyzed by means of elliptic boundary-value problems (see [2] and [3]).

(II) $M$ is a complex manifold and let $\boldsymbol{L}$ be the bundle of tangent vectors of type $(0,1)$ (i.e. locally a section of $\boldsymbol{L}$ is of the form $\sum a^{j} \partial / \partial \bar{z}_{j}$ where $z_{1}, \ldots, z_{n}$ are holomorphic local coordinates). In this example the operator $\mathscr{D}$ is usually denoted by $\bar{\partial}$ and the local solution is given by the so-called Dolbeault lemma. The global problem for compact manifolds can easily be reduced to determined elliptic systems; this is the so-called Hodge theory (see for example [12]). On a manifold with a smooth boundary the global problem is in general not solvable in the sense that the range of $\bar{\partial}$ is not closed in any of the topologies of interest; however, if certain conditions are imposed on the boundary the problem is solvable (see [13] and [7]). Some important questions about the dependence of the solution on $f$ in Hölder norms still remain unanswered although great progress in this area has been made recently (see [11]).

(III) $M$ is a differentiable manifold and $\boldsymbol{L}$ has the property that $\overline{\boldsymbol{L}}=\boldsymbol{L}$ (i.e. locally each $\bar{L}_{k}=\sum \bar{a}_{k}^{j} \partial / \partial x^{j}$ is a linear combination of $L_{1}, \ldots, L_{n}$ ). Then locally by the classical Frobenius theorem we can find local coordinates $x_{1}, \ldots, x_{n}$ such that a local basis for the sections of $\boldsymbol{L}$ over a coordinate 
neighborhood is given by $\partial / \partial x_{1}, \ldots, \partial / \partial x_{m}$ and so the local problem can be settled by an easy adaptation of the solution of example (I). However the global problem is not stable and will not be considered here (see [28]).

(IV) Let $M_{1}$ and $M_{2}$ be manifolds with $L_{1}$ and $L_{2}$ the corresponding subbundles of $C T\left(M_{1}\right)$ and $C T\left(M_{2}\right)$ satisfying conditions $\mathrm{A}$ and $\mathrm{B}$. Let $M=M_{1} \times M_{2}$ and denote by $\pi_{i}: M \rightarrow M_{i}, i=1,2$, the projection maps; these induce maps $\pi_{i}^{*}: C T(M) \rightarrow C T\left(M_{i}\right)$. Now we define $L$ by

$$
L=\left\{L \in C T(M) \mid \pi_{i}^{*} L \in L_{i}, i=1,2\right\} .
$$

This $\boldsymbol{L}$ then satisfies conditions A and B.

(V) Let $M$ be a closed differentiable submanifold of a manifold $M_{1}$. Suppose that $L_{1} \subset C T\left(M_{1}\right)$ satisfies A and B. The identity map $I: M \rightarrow M_{1}$ induces the map $I^{*}: C T(M) \rightarrow C T\left(M_{1}\right)$ and we define

$$
L=\left\{L \in C T(M) \mid I^{*} L \in C T\left(M_{1}\right)\right\} .
$$

Now if we assume that $\boldsymbol{L}$ is a bundle (i.e. that the dimension of the fiber is constant) then we obtain another example. We say that $\boldsymbol{L}$ is the restriction of $L_{1}$ to $M$.

The case, where $M$ is a closed submanifold of a complex manifold $M_{1}$ and $L_{1}$ is as in (II), has been studied extensively (see [20], [18], [29] and [24]). In fact if we take $M_{1}=C^{2}$ and

$$
M=\left\{\left.\left(z_{1}, z_{2}\right) \in C\left|\operatorname{Re} z_{2}=\right| z_{1}\right|^{2}\right\},
$$

setting $t=\operatorname{Im} z_{2}$ and $z=z_{1}$ we find that $z, t$ are local coordinates in the real three-dimensional manifold $M$. In terms of these coordinates the equation (1.12) becomes

$$
\frac{\partial u}{\partial \bar{z}}+i z \frac{\partial u}{\partial t}=f
$$

This is the famous equation of H. Lewy which in general has no solution (see [21]). The discovery of this equation has led to several important developments in partial differential equations (see [9] and [26]). The case where $M=\left\{z \in C^{n}|| z \mid=1\right\}$ has been studied in great detail by G. B. Folland (see [4]).

Condition $\mathrm{C}$. We say that the subbundle $L \subset C T$ satisfies this condition if $\boldsymbol{L} \wedge \overline{\boldsymbol{L}}$ is also a subbundle, i.e. if the fibers are of constant dimension.

It is now natural to ask whether any $L \subset C T$ satisfying conditions $A, B$ and $\mathrm{C}$ can be realized locally performing the constructions in IV and $\mathrm{V}$ on the examples II and III. In case $\boldsymbol{L}$ is one dimensional this reduces to a problem of H. Lewy; namely, given a single nonvanishing complex vector field $L$ do there exist nontrivial solutions of $L u=0$. In case $L$ is real analytic (i.e. there exist coordinates such that the real and imaginary parts of the $a_{k}^{j}$ in (1.1) are analytic) the conjecture is answered affirmatively by the 
Cauchy-Kowalevsky method (see [1] where it is also shown that if coordinates $x_{1}, \ldots, x_{n}$ are chosen so that $\partial / \partial x_{1}, \ldots, \partial / \partial x_{p}$ span the local sections of $\boldsymbol{L} \cap \overline{\boldsymbol{L}}$ then it suffices to assume analyticity in the variables $x_{p+1}, \ldots, x_{n}$ ). Another example in which the above conjecture is established is the case when the sections of $\boldsymbol{L}+\overline{\boldsymbol{L}}$ are closed under the Lie bracket, the result is known as the Nirenberg-Frobenius theorem (see [23], [25] and [8]).

3. The Laplacian and the energy form. We will assume that $L \subset C T(M)$ satisfies conditions A, B and C. Denote by $n$ the dimension of $M, m$ the fiber dimension of $\boldsymbol{L}$ and $p$ the fiber dimension of $\boldsymbol{L} \cap \overline{\boldsymbol{L}}$. Let $\boldsymbol{L}_{0}$ be a subbundle of $\boldsymbol{L}$ such that

$$
L=L_{0} \oplus L \cap \bar{L}
$$

and let $N$ be a subbundle of $C T$ such that

$$
C T=N \oplus(L+\bar{L}) \text {. }
$$

Then we have

$$
C T=N \oplus L_{0} \oplus \bar{L}_{0} \oplus L \cap L .
$$

Choose hermitian metrics on $\boldsymbol{N}, \boldsymbol{L}_{0}$ and $\boldsymbol{L} \cap \overline{\boldsymbol{L}}$; we define a hermitian metric on $\bar{L}_{0}$ by setting

$$
\langle u, v\rangle=\overline{\langle\bar{u}, \bar{v}\rangle}
$$

where $u, v$ are tangent vectors (at the same point) lying in $\overline{\boldsymbol{L}}_{0}$; thus $\bar{u}, \bar{v} \in \boldsymbol{L}_{0}$ and so the right-hand side has meaning. Now we define a hermitian metric on $C T$ by making the direct sum in (3.3) orthogonal-this induces a hermitian metric on $C T^{*}$, on $L^{*}$ and on $L^{*} \wedge L^{*}$. Assume that $M$ has a volume element $d V$. We define the $L_{2}$-inner product on the sections of these bundles by setting

$$
(\varphi, \psi)=\int_{M}\langle\varphi, \psi\rangle d V
$$

Thus we have the adjoint of (1.14) (with $V=M$ ) which is the differential complex

$$
\Gamma\left(L^{*} \wedge L^{*}, M\right) \stackrel{\mathscr{g}^{*}}{\rightarrow} \Gamma\left(L^{*}, M\right) \stackrel{\mathscr{g}^{*}}{\rightarrow} C^{\infty}(M)
$$

where the $\mathscr{D}^{*}$ are the formal adjoints of the $\mathscr{D}$ determined by requiring that

$$
\left(\mathscr{D}^{*} \varphi, \psi\right)=(\varphi, \mathscr{D} \psi)
$$

where $\varphi \in \Gamma\left(L^{*} \wedge L^{*}, M\right)$ and $\psi \in \Gamma\left(L^{*}, M\right)$ and $\psi$ has compact support. The map $\mathscr{D}^{*}: \Gamma\left(L^{*}, M\right) \rightarrow C^{\infty}(M)$ is defined similarly and thus we have

$$
\left(\mathscr{D}^{*}\right)^{2}=0 .
$$


We now define the map $\mathscr{L}: \Gamma\left(L^{*}, M\right) \rightarrow \Gamma\left(L^{*}, M\right)$ by setting

$$
\mathscr{L} \varphi=\mathscr{D}^{*} \varphi+\mathscr{D}^{*} \mathscr{D} \varphi .
$$

$\mathscr{L}$ is called the Laplacian of the complex (1.14), in the case of example (I) this is the usual Laplace-Beltrami operator on 1-forms.

Suppose that $M$ is compact. Now observe that given $f \in \Gamma\left(L^{*}, M\right)$ if we wish to solve the equation (1.12), then $f$ must satisfy not only (1.16) but also, $f$ has to be orthogonal to $\mathscr{N}\left(\mathscr{D}^{*}\right)$, the null space of $\mathscr{D}^{*}$. Since if $\psi \in \mathscr{N}\left(\mathscr{D}^{*}\right)$ we have

$$
(f, \psi)=(\mathscr{D} u, \psi)=\left(u, \mathscr{D}^{*} \psi\right)=0 .
$$

We denote by $\mathscr{H}$ the null space of $\mathscr{L}$. It then follows that

$$
\mathscr{H}=\left\{\varphi \in \Gamma\left(L^{*}, M\right) \mid \mathscr{D} \varphi=0 \text { and } \mathscr{D}^{*} \varphi=0\right\}
$$

since

$$
(\mathscr{L} \varphi, \varphi)=\|\mathscr{D} \varphi\|^{2}+\left\|\mathscr{D}^{*} \varphi\right\|^{2},
$$

and we obtain:

3.13 Lemma. Given $f \in \Gamma\left(M, L^{*}\right)$ if there exists $u \in C^{\infty}(M)$ satisfying (1.12) then $f$ satisfies (1.16) and is orthogonal to $\mathscr{H}$. Furthermore the conditions (1.16) and orthogonality to $\mathscr{H}$ are equivalent to (1.16) and orthogonality to $\mathscr{N}\left(\mathscr{D}^{*}\right)$.

Consider the equation

$$
\mathscr{L} \varphi=f,
$$

where $\varphi, f \in T\left(M, L^{*}\right)$ it is clear that $f \perp \mathscr{H}$, as in (3.10), since $\mathscr{L}^{*}=\mathscr{L}$.

3.15 Proposition. Suppose that for every $f \in \Gamma\left(M, L^{*}\right)$ with $f \perp \mathscr{H}$ there exists a $\varphi \in \Gamma\left(M, L^{*}\right)$ satisfying (3.14), then for every $f$ which satisfies the necessary conditions given in 3.13 there exists a $u \in C^{\infty}(M)$ such that $\mathscr{D} u=f$.

Proof. We have $f \perp \mathscr{H}$ and $\mathscr{D} f=0$, furthermore

$$
f=\mathscr{D}^{*} \varphi+\mathscr{D}^{*} \mathscr{D} \varphi .
$$

We will show that $\mathscr{D}^{*} \mathscr{D} \varphi=0$ and then setting $u=\mathscr{D}^{*} \varphi$ will complete the proof. Since $\mathscr{D} f=0$ and $\mathscr{D}^{2}=0$ we conclude that

$$
\mathscr{D}^{*} \mathscr{D} \varphi=0 \text {. }
$$

Taking inner products into $\mathscr{D} \varphi$,

$$
\left(\mathscr{D} \mathscr{D}^{*} \mathscr{D} \varphi, \mathscr{D} \varphi\right)=\left\|\mathscr{D}^{*} \mathscr{D} \varphi\right\|^{2}=0
$$

and hence $\mathscr{D}^{*} \mathscr{D} \varphi=0$ as required. 
Thus the global problem of solving the over-determined system (1.12) on a compact manifold is reduced to solving the determined system (3.14). To study the operator $\mathscr{L}$ we introduce the hermitian form $Q: \Gamma\left(L^{*}, M\right)$ $\times \Gamma\left(L^{*}, M\right) \rightarrow C$ defined by

$$
Q(\varphi, \psi)=(\mathscr{D} \varphi, \mathscr{D} \psi)+\left(\mathscr{D}^{*} \varphi, \mathscr{D}^{*} \psi\right)+(\varphi, \psi) .
$$

There is a general theorem which asserts that if $Q$ is compact with respect to $L_{2}$ (i.e. if a sequence $\varphi_{v} \in \Gamma\left(L^{*}, M\right)$ is bounded in the sense $Q\left(\varphi_{v}, \varphi_{v}\right)<C$ then it has a subsequence that converges in $\left.L_{2}\right)$, then the space $\mathscr{H}$ is finite dimensional and the system (3.14) has a unique solution in $\Gamma\left(L^{*}, M\right)$ which is orthogonal to $\mathscr{H}$ (see [16]). An example of W. Sweeney (see [31]) shows that there are differential complexes for which the energy form is not compact and whose Laplacian, nevertheless, can be inverted. Here, however, we will discuss the problem of finding circumstances under which $Q$ is compact.

4. Hypoellipticity and the case of co-dimension one. To show that $Q$ is compact (and that $\mathscr{L}$ is hypoelliptic) it suffices to show that every point of $M$ has a neighborhood $U$ on which there are vector fields $Z_{1}, \ldots, Z_{q}$ such that the Lie algebra generated by these equals $\Gamma(C T, U)$ and that there exists $C>0$ such that

$$
\sum_{r, s}\left\|Z_{r} \varphi_{s}\right\|^{2} \leqq C Q(\varphi, \varphi)
$$

for all $\varphi \in \Gamma\left(L^{*}, U\right)$ with compact support, here $\varphi_{s}$ denote the components of $\varphi$ relative to some fixed local basis of $\Gamma\left(L^{*}, U\right)$. The special case where the $Z$ 's and single Lie brackets span all tangent vector fields was proved by the author (see [14]); the general case was proved by Hörmander (see [10], a proof based on pseudo-differential operators was found independently by Radkevitch [27] and the author [17]).

Let $L_{1}, \ldots, L_{m-p}$ be a local orthonormal basis of $\Gamma\left(\boldsymbol{L}_{0}, U\right)$, where $U$ is a small neighborhood in $M$, and let $A_{1}, \ldots, A_{p}$ and $N_{1}, \ldots, N_{n+p-2 m}$ be local orthogonal bases of $\Gamma(\boldsymbol{L} \cap \overline{\boldsymbol{L}}, U)$ and $\Gamma(N, U)$ respectively. Thus $L_{1}, \ldots, L_{m-p}, \bar{L}_{1}, \ldots, \bar{L}_{m-p}, A_{1}, \ldots, A_{p}, N_{1}, \ldots, N_{n+p-2 m}$ is a local basis for the tangent vector fields on $U$. Let $\omega^{\prime}, \ldots, \omega^{m-p}, \bar{\omega}^{\prime}, \ldots, \bar{\omega}^{m-p}$, $\alpha^{1}, \ldots, \alpha^{p}, \eta^{\prime}, \ldots, \eta^{n+p-2 m}$ be the corresponding local basis of the 1 -forms. Then if $\varphi \in \Gamma\left(L^{*}, U\right)$ we can write

$$
\varphi=\sum_{j=1}^{m-p} \varphi_{j} \omega^{j}+\sum_{j=1}^{p} \varphi_{j+m-p} \alpha^{j}
$$

and we have

$$
\mathscr{D} u=\sum_{j=1}^{m-p}\left(L_{j} u\right) \omega^{j}+\sum_{j=1}^{p}\left(A_{j} u\right) \alpha^{j}
$$


Further a simple calculation shows that if

$$
\mathscr{L} \varphi=\sum_{k=1}^{m-1}(\mathscr{L} \varphi)_{k} \omega^{k}+\sum_{k=1}^{p}(\mathscr{L} \varphi)_{k+m-p} \alpha^{k}
$$

then

$$
\begin{aligned}
(\mathscr{L} \varphi)_{k}= & -\left(\sum_{j=1}^{m-p} \bar{L}_{j} L_{j}+\sum_{j=1}^{p} A_{j}^{2}\right) \varphi_{k}+\sum_{i=1}^{m-p}\left[\bar{L}_{i}, L_{k}\right] \varphi_{i} \\
& + \text { combinations of the } L, \bar{L} \text { and } A, \\
& \text { when } k=1, \ldots, m-p,
\end{aligned}
$$

and

$$
\begin{aligned}
(\mathscr{L} \varphi)_{k}= & -\left(\sum_{j=1}^{m-p} \bar{L}_{j} L_{j}+\sum_{j=1}^{p} A_{j}^{2}\right) \varphi_{k} \\
& + \text { combinations of the } L, \bar{L} \text { and } A, \\
& \text { when } k=m-p+1, \ldots, m .
\end{aligned}
$$

Note that the dimension of the fiber of $\boldsymbol{L}+\overline{\boldsymbol{L}}$ is $2 m-p$ and that the dimension of the fiber of $\boldsymbol{N}$ equals the co-dimension of $\boldsymbol{L}+\overline{\boldsymbol{L}}$ equals $n+p-2 m$. Suppose that the co-dimension is one. We choose $N_{1}=N$ such that $N=-\bar{N}$. Then we have

$$
\left[\bar{L}_{i}, L_{k}\right]=c_{i k} N+\text { combinations of the } L, \bar{L} \text { and } A \text {. }
$$

In the case where the $\boldsymbol{L}$ is obtained on a submanifold of a complex manifold of real co-dimension one the $\left(c_{i k}\right)$ is the Levi-form.

4.6 THEOREM. If the bundle $\boldsymbol{L} \subset \boldsymbol{C T}$ satisfies conditions A, B and $\mathrm{C}$ and if the fibers of $\boldsymbol{L}+\overline{\boldsymbol{L}}$ have co-dimension one, then the following are equivalent: (a) each point has a neighborhood $U$ such that there exist $C>0$ such that

$$
\sum_{j, k}\left\|L_{j} \varphi_{k}\right\|^{2}+\sum_{j, k}\left\|\bar{L}_{j} \varphi_{k}\right\|^{2}+\sum_{j, k}\left\|A_{j} \varphi_{k}\right\|^{2} \leqq C Q(\varphi, \varphi)
$$

for all $\varphi \in \Gamma\left(L^{*}, U\right)$ with compact support; and (b) $m \geqq 2$ and the matrix $\left(c_{i k}\right)$ has either all eigenvalues of the same sign or has two pairs of eigenvalues of opposite sign.

The proof of this theorem can be obtained by an approximate "uncoupling" of the above system. For a fixed point $P \in M$ we choose the orthogonal local basis $L_{1}, \ldots, L_{m-p}$ such that $c_{i k}(P)=\delta_{i k} \lambda_{k}$, where the $\left(c_{i k}\right)$ are given 
by (4.5). Now we define the differential operators $P_{k}: C^{\infty}(U) \rightarrow C^{\infty}(U)$, $k=1, \ldots, m-p$, by

$$
P_{k} u=-\left(\sum_{j=1}^{m-p} \bar{L}_{j} L_{j}+\sum_{j=1}^{p} A_{j}^{2}\right) u+\lambda_{k} N u .
$$

4.8 THEOREM. The following are equivalent:

(a) There exists $C>0$ such that

$$
\operatorname{Re}\left(P_{k} u, u\right)+\|u\|^{2} \geqq C\left(\sum_{j=1}^{m-p}\left\|L_{j} u\right\|^{2}+\sum_{j=1}^{m-p}\left\|\bar{L}_{j} u\right\|^{2}+\sum_{j=1}^{p}\left\|A_{j} u\right\|\right)^{2}
$$

for all $u \in C_{0}^{\infty}(U)$.

(b) Either $\lambda_{k} \neq 0$ and there is another eigenvalue of the same sign or $\lambda_{k}=0$ and there are two nonzero eigenvalues of opposite sign.

It then follows that if $U$ is sufficiently small and if (4.9) holds for $k=1, \ldots, m-p$ then (4.7) also holds.

For the proof of this see [15].

The imaginary part of the first order terms in the operators $P_{k}$ plays a vital role here, so that they do not fall under the theory developed by Hörmander and Radkevitch (see [10] and [27]) in which the subprincipal part is real. A similar phenomenon as that presented by the operators $P_{k}$ is found in the work of Grushin (see [6]). He considers special types of equations in $\boldsymbol{R}^{n}$ with polynomial coefficients which are elliptic outside of a linear subspace. One of his examples is as follows:

$$
P u=\frac{\partial^{2} u}{\partial x_{n}^{2}}+x_{n}^{2} \sum_{j=1}^{n-1} \frac{\partial^{2} u}{\partial x_{j}}+i \theta \frac{\partial u}{\partial x_{1}}
$$

where $\theta \in \boldsymbol{C}$. In [6] the following is proved:

4.11 Proposition. $P$ is hypoelliptic if and only if either $\operatorname{Im} \theta \neq 0$ or $\operatorname{Im} \theta$ $=0$ and $|\theta|<1$ when $n>2$ or $\operatorname{Im} \theta=0$ and $\theta$ does not equal an odd integer.

Using the methods above, part of this proposition can be generalized as follows (see [15]).

4.12 Proposition. If $X_{1}, \ldots, X_{k}$ are real vector fields defined on a neighborhood $U \subset \boldsymbol{R}^{n}$ and if the $\left\{X_{j}\right\}$ and $\left[X_{i}, X_{j}\right]$ span all the tangent vector fields then the operator

$$
P u=-\sum_{j=1}^{k} X_{j}^{2} u+i \theta\left[X_{1}, X_{2}\right] u+c u
$$

where $\theta \in C$ and $c \in C^{\infty}(U)$ is hypoelliptic whenever $\operatorname{Im} \theta \neq 0$ and whenever $\operatorname{Im} \theta=0$ and $|\theta|<1$. In fact these conditions are necessary and sufficient for the estimate 


$$
\operatorname{Re}(P u, u)+\|u\|^{2} \geqq c \sum_{j=1}^{k}\left\|X_{j} u\right\|^{2}
$$

for all $u \in C_{0}^{\infty}(U)$ with support in a fixed compact subset of $U$.

5. The local problem. The local problem is considerably more difficult than the global one (on compact manifolds). This can be seen already in the case of integrable almost complex structure where global existence is obtained from standard elliptic theory, but the local theorem depends on the Newlander-Nirenberg theorem. Now there are three distinct proofs of the Newlander-Nirenberg theorem, the original proof (see [23]) and the proofs given in [13] and [22]. The original proof depends heavily on the Cauchy formula so it seems more promising to generalize the latter two. To generalize the proofs of [13] one needs to understand the restriction of $L$ to the boundary - this presents two difficulties : first that the higher co-dimension case is not yet understood, and second that the restriction will in general not satisfy condition B. There is some hope that these difficulties can be overcome, possibly by developing methods analogous to those in [19]. Malgrange's method of proof depends on the fact that solutions of analytic elliptic equations are analytic; it is possible that this is also true for the corresponding equations obtained from our problems. Recent results of Treves (see [32]) indicate that this may be so.

In conclusion we remark that very general overdetermined systems can be reduced, using the Spencer resolution (see [30]) to the ones discussed here (see also [5]).

\section{REFERENCES}

1. A. Andreotti and C. D. Hill, Complex characteristic coordinates and tangential CauchyRiemann equations, Ann. Scuola Norm. Sup. Pisa (to appear).

2. P. E. Conner, The Neumann's problem for differential forms on Riemannian manifolds, Mem. Amer. Math. Soc. No. 20 (1956). MR 17, 1197.

3. K. O. Friedrichs, Differential forms on Riemannian manifolds, Comm. Pure Appl. Math. 8 (1955), 551-590. MR 19, 407.

4. G. B. Folland, Tangential Cauchy-Riemann complex on a sphere in $C^{n}$, Doctoral Dissertation, Princeton University, Princeton, N.J., 1971.

5. V. Guillemin and S. Sternberg, Subelliptic estimates for complexes, Proc. Nat. Acad. Sci. U.S.A. 67 (1970), 271-274.

6. V. V. Grušin, On a class of hypoelliptic operators, Mat. Sb. 83 (125) (1970), 456-473= Math. USSR Sb. 12 (1970), 458-476.

7. L. Hörmander, An introduction to complex analysis in several variables, Van Nostrand, Princeton, N.J., 1966. MR 34 \# 2933.

8. ㄴ, The Frobenius-Nirenberg theorem, Ark. Mat. 5(1965), 425-432. MR 31 \# 2480.

9. - Differential equations without solutions, Math. Ann. 140 (1960), 169-173. MR 26 \# 5279.

10. - Hypoelliptic second order differential equations, Acta Math. 119 (1967), 147-171. MR 36 \#5526.

11. N. Kerzman, Hölder and $L^{p}$ estimates for solutions $\partial u=f$ in strongly pseudoconvex domains, Comm. Pure Appl. Math. 24 (1971), 301-379.

12. K. Kodaira, Harmonic fields in Riemannian manifolds (generalized potential theory), Ann. of Math. (2) $\mathbf{5 0}$ (1949), 587-665. MR 11, 108. 
13. J. J. Kohn, Harmonic integrals on strongly pseudo-convex manifold. I, II, Ann. of Math. (2) 78 (1963), 112-148; ibid. (2) 79 (1964), 450-472. MR 27 \# 2999; 34 \# 8010.

14. - Boundaries of complex manifolds, Proc. Conf. Complex Analysis (Minneapolis, Minn., 1964), Springer, Berlin, 1965, pp. 81-94. MR 30 \# 5334.

15. Complex hypoelliptic equations, Proc. Conf. on Hypo-Elliptic Operators, Rome, 1971.

16. J. J. Kohn and L. Nirenberg, Non-coercive boundary value problems, Comm. Pure Appl. Math. 18 (1965), 443-492. MR 31 \#6041.

17. J. J. Kohn, Pseudo-differential operators and non-elliptic problems, Pseudo-Differential Operators (C.I.M.E., Stresa, 1968), Edizioni Cremonese, Rome, 1969, pp. 157-165. MR 41 \# 3972.

18. J. J. Kohn and H. Rossi, On the extension of holomorphic functions from the boundary of a complex manifold, Ann. of Math. (2) 81 (1965), 451-472. MR 31 \# 1399.

19. M. Kuranishi, Convexity conditions related to $\frac{1}{2}$-estimates in elliptic complexes, Proc. Internat. Congress of Math., Nice, 1970.

20. $\mathrm{H}$. Lewy, On the local character of the solutions of an atypical linear differential equation in three variables and a related theorem for regular functions of two complex variables, Ann. of Math. (2) 64 (1956), 514-522. MR 18, 473.

21. - An example of a smooth linear partial differential equation without solution, Ann. of Math. (2) 66 (1957), 155-158. MR 19, 551

22. B. Malgrange, Pseudo-groupes de Lie elliptiques, Séminaire les équation aux derivées parielles I, College de France 1969-1970.

23. A. Newlander and L. Nirenberg, Complex analytic coordinates in almost complex manifolds, Ann. of Math. (2) 65 (1957), 391-404. MR 19, 577.

24. R. Nirenberg, On the H. Lewy extension phenomenon, Trans. Amer. Math. Soc. (to appear).

25. - A complex Frobenius theorem, Seminar on Analytic Functions, Institute of Advanced Studies, 1957, pp. 172-189.

26. L. Nirenberg and F. Treves, On local solvability of linear partial differential equations. I: Necessary conditions; II: Sufficient conditions, Comm. Pure Appl. Math. 23 (1970), 1-38, 459-510.

27. E. V. Radkevič, Hypoelliptic operators with multiple characteristics, Mat. Sb. 79 (121) (1969), 193-216 = Math. USSR Sb. 8 (1969), 181-205. MR 41 \# 5763.

28. B. L. Reinhart, Harmonic integrals on almost product manifolds, Trans. Amer. Math. Soc. 88 (1958), 243-276. MR 21 \# 3687.

29. H. Rossi, Differentiable manifolds in complex euclidean space, Proc. Internat. Congress Math. (Moscow, 1966), "Mir”, Moscow, 1968, pp. 512-516; Proc. Nat. Acad. Sci. U.S.A. 67 (1970), 271-274. MR 38 \#2816.

30. D. C. Spencer, Overdetermined systems of linear partial differential equations, Bull. Amer. Math. Soc. 75 (1969), 179-239. MR 39 \# 3533.

31. W. J. Sweeney, A noncompact Dirichlet norm, Proc. Nat. Acad. Sci. U.S.A. 58 (1967), 2193-2195. MR 37 \# 3587.

32. F. Treves, Hypoelliptic partial differential equations of principal type with analytic coefficients, Comm. Pure Appl. Math. 23 (1970), 637-651.

Department of Mathematics, Princeton University, Princeton, New Jersey 08540 